\title{
Performance based Mixed Bias Efficient Algorithms for Wireless Mesh Networks with Multiple Gateway
}

\author{
Shivbhanu Dangi \\ Department of Comp. Science \& Engineering \\ Samrat Ashok Technological Institute \\ Vidisha, (M.P.), India.
}

\author{
Ram Ratan Ahirwal \\ Department of Comp. Science \& Engineering \\ Samrat Ashok Technological Institute \\ Vidisha, (M.P.), India.
}

\begin{abstract}
Wireless Mesh Network (WMN) has become an important edge network to provide Internet access to remote areas and wireless connections in a metropolitan scale. The wireless mesh network and the associated IEEE 802.11s standard have attracted an enormous amount of research in this field from the past few years. In our proposed approach first, we are discussed multiple gateway fair scheduling process which consists of distributed routing and requirement tables and a propagation algorithm for scheduling at the gateways. Then a mixed-bias fair scheduling which is bias against different characteristics of the network. This technique biases against characteristics of the network which are detrimental to performance, fairness, or both.
\end{abstract}

\section{KEYWORDS}

WMN, Scheduling Approach, Multiple Gateway Protocol, and Cross-Layered Mixed-bias Approach.

\section{INTRODUCTION}

WMNs have been identified as key technology to enhance and compliment existing network installations as well as provide access where traditional technology is not available or too costly. A WMN is made up of mesh routers (MRs), which have limited or no mobility, and mesh clients (MCs) which are often fully mobile. The mesh routers form the backbone of the network allowing the clients to have access to the network through the backbone. We propose an algorithm for fair scheduling in WMNs with multiple gateways. We also propose another algorithm for scheduling which places more emphasis on throughput while retaining a basic level of throughput called mixed-bias. This technique biases against characteristics of the network which are detrimental to performance, fairness, or both [1].

WMN is a multi-hop wireless network; there are unique challenges to deal with when compared with traditional wired and wireless networks. The wireless channel is a broadcast medium, meaning that all nodes within a certain range are subjected to interference and cannot transmit simultaneously. At the same time, it is difficult to sense whether communication is taking place in other parts of the network because of hidden and exposed node problems where an intermediate node may be stuck in between two nodes which are trying to transmit simultaneously but out of range of each other. The solution too many of these problems can be scheduling of transmissions. As mentioned previously, scheduling in WMNs seems to be a problem of balancing two often disjoint goals; one is ensuring fairness among client nodes in the network, another is ensuring the network is performing at as close to capacity as possible. Figure 1 is illustrated a wireless mesh network [2].

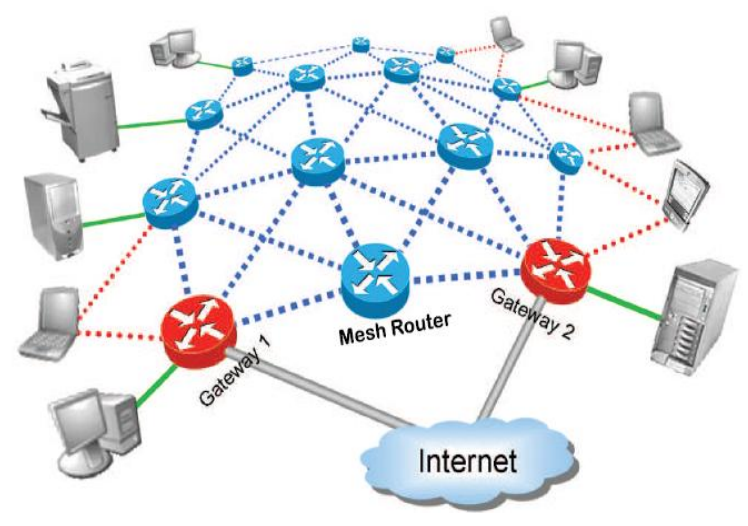

Figure 1 Illustration of a Wireless Mesh Network

The goal of a good scheduling algorithm is to find a balance between these two goals. This paper provide scheduling techniques for wireless mesh networks which find a balance between fairness and throughput in WMNs using mixed bias approach. [1], [2], [3], [4] , [5].

\section{BACKGROUND TECHNIQUES}

The first motivation for studying fairness in WMNs is in networks where users are paying equal amounts of money for service and expect a similar quality of service (QoS). Often existing solutions focus on either the problem of throughput or the problem of fairness. It is often difficult to create a solution which addresses both of these problems since they are divergent goals. Recently, however, it is possible to have high throughput solutions that avoid node starvation. Mesh Clients (MCs) which are far away from the gateways (in terms of hops) often receive much lower QoS than those which are very close. This is because while the farther users' packets are traversing all of the hops along the path, there is a transmission and queuing delay at each hop. The nodes which are close to the gateways do not experience this and can often transmit many packets while the farther nodes are still waiting for one packet to arrive. However, if we give each node enough time to transmit regardless of distance the throughput of the network decreases dramatically. This is because the delay increases greatly by giving each node enough time to transmit regardless of distance to the GW. Some nodes may end up waiting almost indefinitely while other nodes are transmitting [2], [3]. 


\section{CLASSIFICATION OF FAIR SCHEDULING TECHNIQUES}

Fair scheduling protocols for wireless mesh networks can be classified into four categories. These categories in order of fairness from the most fair to the least fair are: Hard-fairness, max-min, proportional fairness, mixed-bias and maximum throughput. This classification and the relationship are among them.

Fair Scheduling Techniques

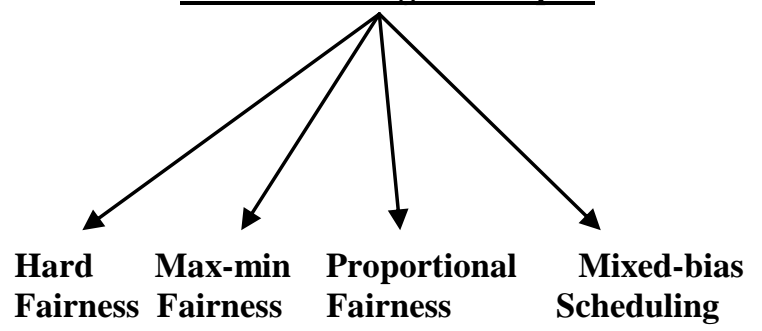

The details descriptions of above fair scheduling techniques are as follow.

\section{Hard Fairness}

Hard fairness is also known as round-robin scheduling. It has been used in some of the earliest wireless networks and in simplistic network models since it is the least complex. It is the fairest scheme since each node is guaranteed exactly equal amount of time in order. In networks where the nodes only require a small proportion of resources hard fairness causes problems. Since each node is given time to transmit at regular intervals, if the node does not have any data to send, the time is wasted. This leads to very low overall throughput [4] and [5].

\section{Max-min fairness}

Max-min fairness allocates resources in order of increasing demand. The minimum amount of resources assigned to each node is maximized. So if there are more than enough resources for each node, every node gets what it needs. If there is not, the resources are split evenly. This means that the nodes which require fewer resources get a higher proportion of their need satisfied. The nodes which require more resources end up dropping many packets and thus the network ends up with still quite low packet delivery ratio. This type of scheme works best in situations where there is not large differences in resources requested at each node. This can be a problem in a mesh network because intuitively, the nodes closer to the gateways will experience much higher traffic than those on the outside of the network, yet may end up dropping many of the packets anyway. This may be partially solved by increasing the resource capacity of nodes closest to the gateways [6] and [7].

\section{Proportional fairness}

Proportional fairness allocates resources proportional to some characteristic in the network. For example, one may choose to give priority to nodes which are close to the gateways in a wireless mesh network. The amount of resources allocated then would be proportional to how close the node is to the gateway.

\section{Mixed-bias Scheduling}

Mixed-bias scheduling allows for different levels of control over resources. Rather than just allowing for one bias, this scheme mixes two different biasing levels together. A certain proportion of the resources are assigned to one factor and the rest to another factor. This allows the scheduling algorithm to provide two different biasing levels or "mixed-biasing" against a certain characteristic. Rather than just strongly biasing against that characteristic which may result in certain nodes to be starved, the mixed-biasing allows for a combination of weak and strong biasing meaning that a portion of the resources are reserved to provide a minimum service level, even for the nodes which are undesirable in terms of certain characteristics [8-9].

\section{RELATED WORKS}

Nessrine Chakchouk and Bechir Hamdaoui et. al. proposed scheme is interference and traffic aware in that it increases the overall achievable throughput of the network by eliminating the interference between the wireless mesh routers and maximizes the satisfaction ratios of all active sessions by accounting for the sessions' data rate requirements. Simulation results show that the proposed scheme outperforms the Tabu-based scheduling scheme and yields good tradeoffs between the achievable throughput of the network and the satisfaction ratios of the sessions. [1].

Erwu Liu, and Qinqing Zhang and Kin K. Leung et. al. provided a clique-based method with efficient spatial reuse, which is then incorporated into proportionally fair scheduling (PFS) for fair resource management in WMNs. We call it a clique-based proportionally fair scheduling (CBPFS) algorithm. [2].

P.Saravanaselvi and P.Latha et. al. showed the performance of Weighted Fair Queuing (WFQ) scheduling algorithm is evaluated to attain Quality of Service (QoS). The weight is assigned for a different service depends on the percentage of bandwidth utilization and priority of services. The proposed scheduling algorithm has designed and simulated using MatLab. The performance is evaluated with the other existing scheduling algorithm like First In First Out (FIFO), Priority queuing (PQ) and Weighted Round Robin (WRR). The behavior of Weighted Fair Queuing scheduling algorithms in WiMAX has been investigated in this work.[3].

Ronghui Hou, King-Shan Lui, Fred Baker, and Jiandong Li provided a hop by hop routing with bandwidth guarantees. In this paper, they study the problem of identifying the maximum available bandwidth path, a fundamental issue in supporting quality-ofservice in WMNs. Due to interference among links, bandwidth, a well-known bottleneck metric in wired networks, is neither concave nor additive in wireless networks. They propose a new path weight which captures the available path bandwidth information. They formally prove that their hop-by-hop routing protocol based on the new path weight satisfies the consistency and loop-freeness requirements [4]

Yongsu Gwak, Jinsoo Ahn and Young Yong Kim propose bridge routing based on network coding for wireless mesh network Bridge routing offers the solution to exploit the network coding to minimize the usage of time slot. We present feasible and practical ways to study the performance of routing with network coding.[5]

Jad El-Najjar, Hamed M.K. AlAzemi, and Chadi Assi studies the interplay between network coding and spatial reuse in wireless mesh networks. They present a method that attempts to maximize the system performance by exploiting effectively (and not greedily) coding opportunities through appropriate routing and achieving efficient spectrum reuse through opportunistic link scheduling. [7]

\section{PROPOSED WORK}

In this work, we present two approaches for scheduling in WMNs. The first approach is the fair scheduling approach. The emphasis of 
this approach is fairness, leaving overall throughput as a secondary goal. This is especially important when trying to provide equal service to all users within the WMN. However, this approach is not always the best for maximizing throughput and can waste some of the bandwidth in the network. As an alternative to this approach we also present the mixed-bias scheduling approach. This approach is a unique interpretation of the mixed-bias technique except instead of biasing against just one characteristic of the network like the original work, we propose to bias against several characteristics so that a balance between throughput and fairness will be provided to all users.

The first main contribution of this paper is "A fair scheduling for WMNs with multiple gateways". The individual algorithms and components which make up this scheme are outlined in detail. These components include the distributed requirements table, the requirements propagation algorithm. The details description of this algorithm is given below. In the second, we discuss in detail the mixed-bias approach in general. This will then be broken down into each of the mixed-bias techniques that will be combined in the second main contribution of this paper: the combined mixed-bias scheduling approach. In this approach three of the mixed-bias techniques are discusses which biases against several characteristics which are detrimental to network performance and fairness.

\section{Fair scheduling for WMNs with multiple gateways}

In this scheme, the approach is made up of several different components. Each of these components will be outlined in greater detail in the following subsections. This section will provide a general outline of the fair scheduling approach with multiple gateways, highlighting the main contributions we have made to this approach. The original work proposed only a scheduling, however, does not provide a mechanism for maintaining and collecting requirements. The requirements are required for generating the scheduling since this information tells how busy each link is. Thus we propose a distributed manner of accomplishing this. Each mesh router keeps track of a local requirement table. In this requirement table, the demand on each link between the router and a neighbor is kept. When a new schedule is requested, each gateway asks for the partial requirement tables from each mesh router associated with it. The gateway then combines these tables to form one complete requirement table which it uses to generate cliques and eventually the scheduling. One main difference of this approach is that we assume multiple gateways. This means that each gateway in the network is responsible for scheduling all of the links which will forward packets towards it. The single gateway assumption is a significant one for two reasons:

1. The single gateway causes an extreme bottleneck in the network. All traffic which flows in and out of the network must use this node and so any scheduling work done in the network is limited by the single gateway.

2. Similarly, the single gateway node causes a single point of failure in the network. If the gateway node is to go down in this scheme, there is no recovery.

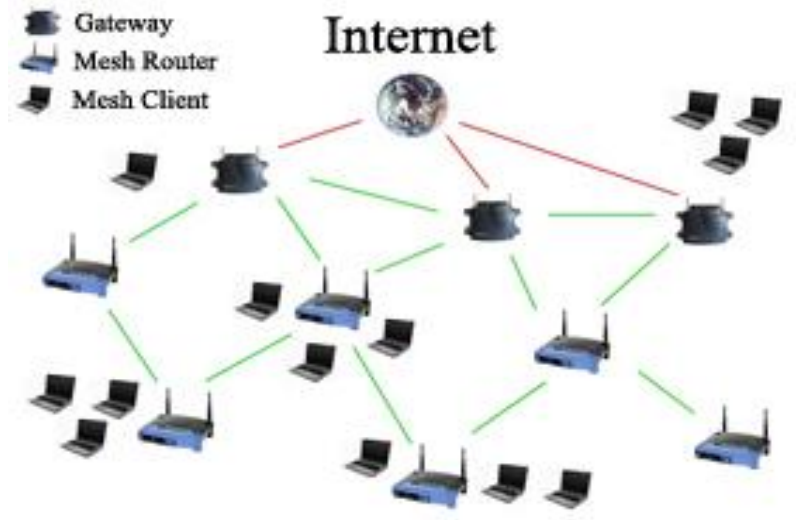

Figure 2 Wireless Mesh Network with multiple gateways.

When multiple gateways are assumed, the bottleneck is eliminated. Not all of the traffic is destined to the same node in the network and is spread more evenly, especially with strategic gateway placement. With a more complex scheme than we proposed, one could further take advantage of the multiple gateways and perform load balancing on the multiple gateways so that under-utilized gateways could be taken advantage of for further performance improvements. Lastly, the single point of failure is eliminated as well. If one gateway experiences an outage, the network has the ability to reconfigure itself to forward packets and perform scheduling from another gateway. Once the requirement table is formed, the gateway uses this information along with the clique information to form a scheduling plan. The clique information is all of the sets of links which may transmit at the same time without interfering with one-another. The clique information is generated once before any transmissions occur in the network in a manner similar to the way neighbors'. In our system model we assume static nodes and topology, so no nodes are added or removed and there is no mobility. Thus we do not need to generate this information more than once in the life of the simulation. This is important because this operation is very expensive computationally, because clique enumeration is known to be a difficult problem to compute. If we were to assume non-static topology, we may have to make an assumption of a certain network size based on the computational resources of the gateway nodes in the network. Using both the clique information and the requirement information, we can then determine which links should be activated together and for how long. A further modification of this scheme would be to use different characteristics other than demand on a link, for example the quality of the link and the distance from the gateway could also be taken into account using a biasing scheme as we have proposed.

\section{Requirement propagation Algorithm}

We proposed the requirement propagation algorithm which allows each gateway to distribute the requirements and routing table for the scheduling into the network. At each mesh router, the path to the gateway is maintained. In this table, requirements for the links on this path are also maintained. For each client requesting to use this mesh router, each link along the way to the gateway in the local table is given a requirement. When the gateway signals the start time for new schedule generation, it requests the local requirement information from all of the mesh routers which are currently using it as their primary gateway.

The requirement propagation algorithm given in Algorithm 1, allows the gateway to keep track of the requirements across all of the links. At the MR, a table containing a partial representation of the network is kept for all of the MRs on the way to the gateway. 
When a MC associates with a given MR, the requirement is incremented for all the MRs along the way to the gateway in the local table. When a new schedule generation is to be completed, the GW requests for the requirements from all of the MRs and combines the results from the partial tables to determine which links must be activated and for how long.

\section{Requirement table used in the gateway node and mobile router node}

\begin{tabular}{|c|c|c|c|}
\hline Dest. & $\begin{array}{l}\text { Next } \\
\text { Hop }\end{array}$ & $\begin{array}{c}\text { Metric } \\
\text { (Hops) }\end{array}$ & $\begin{array}{c}\text { Seq. } \\
\text { No. }\end{array}$ \\
\hline & & & \\
\hline & & & \\
\hline & & & \\
\hline
\end{tabular}

The above table contains the shortest path to every possible destination in the network and number of hops to the destination. A new broadcast route contains

--Destination Address

--Number of hops to reach the destination

--Sequence number of the information about the destination and a new sequence number unique to broadcast.

\section{Requirement Propagation Algorithm Pseudo code:}
1. MC Associate with MR for network use.
2. Generate a Mobile Client Requirement at Mobile routers.
3. For each link between $M R$ and $G W$
- Requirement(current-link) ++
4. Update the requirement table at the gateway node.
5. On Drop: For each link between MR and GW
- Requirement(current-link) --

\section{Algorithm 1 Requirement Propagation}

In this scheme, each gateway is responsible for generating the centralized scheduling for all of the links routing to it. The distribution and coordination of the scheduling is done through the use of START and $E(D$ packets. The gateway sends a START packet to the MR when it has scheduled time to send and an $E(D$ packet when it no longer has permission. It is assumed that these control packets are sent on a different channel from the data and thus do not interfere with data traffic. At the end of one cycle of scheduling, the process is repeated with a new scheduling plan being computed and distributed throughout the network.

The round-robin nature of the scheduling allows the solution to be simple compared to techniques that include weighting functions. At the same time, when compared with a naive round-robin technique, less time is wasted waiting for links which have no traffic to send since time is only allocated to links with requirements. Since we are concerned with fairness among clients who are paying similarly for equal service, this solution works well. Many existing solutions make use of similar round-robin style techniques but none of them use of multiple gateways. Using a single GW to serve a large mesh network is impractical; however, since it becomes a bottleneck quickly as the network size grows.

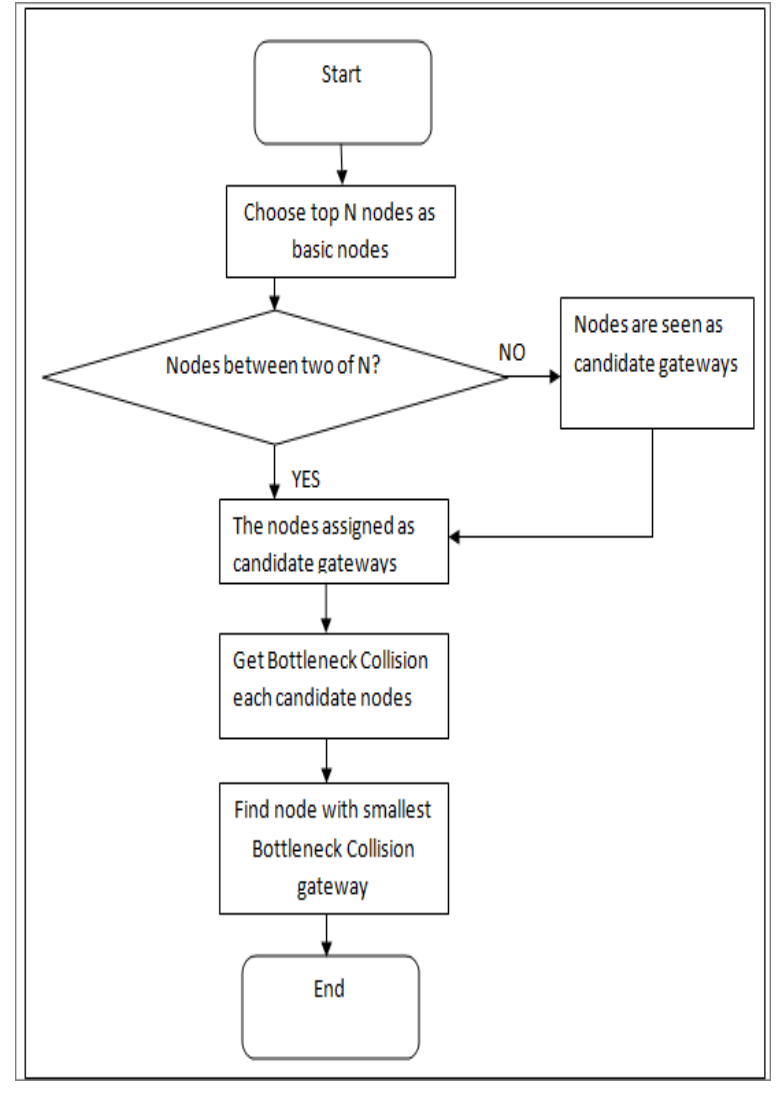

Figure: 3 The flow chart of gateway Scheduling

\section{Mixed-bias Techniques with different characteristic of the network.}

There are four main techniques we present in the following crosslayer mixed-bias.

The first technique uses mixed-biasing to bias against the distance from the gateways. This technique is important because the farther away from the GW a MR is, the more hops the packet must traverse to arrive at the GW. This means the probability of a successful delivery decreases as the MRs are farther away from the $\mathrm{GW}$. Also the average delay increases. Thus if we allow fewer packets from the farthest gateways will achieve higher throughput overall. At the same time, as the packets move closer to the GW successfully, there is a greater chance they will arrive since the closer MRs are given preference.

The second technique favors MRs which has full queues, and thus biases against those with empty or near-empty queues. This is important because if we can give some preference to these routers, perhaps fewer packets will be dropped by reducing the frequency with which the queue is full. By giving preference to full queues, we let the near empty queues build up and at the same time, allow the extremely full queues to empty. This results in a balancing of all the queues in the network. If we can reduce the number of dropped packets, the delay will likely also fall significantly since the overhead in resending a packet is often quite large. This is especially true in multi-hop networks such as WMNs since the retransmission control packets must also traverse the multiple hops.

The third technique biases against poor links. This is important because link quality may change often depending on objects blocking signals, environmental conditions (such as weather, temperature etc) that may make certain links in the network 
perform better than others. When we bias against these links, we give preference to those links which are performing well and allow them to transmit more, thus increasing the overall packet delivery ratio.

In our system model we are applying a similar technique to a WMN which has random source and one gateway as a destination. This is different from the original approach because we introduce the concept of a gateway making the network model closer to that of WMN and less like a wireless ad hoc network. We chose to use the same biasing parameters for our system; however, this could all be altered resulting in vastly different results. Experimenting with different parameters could be left up to the network administrator since it is more dependent on the environment the network resides in and the applications which are being run on it. We include analysis of the technique presented as a verification of their results, and as a benchmark for our own schemes. Equation 1 shows the mixed-bias equation which is used to assign resources to a given node based on its distance from the gateway it is routing to:

$$
R=\frac{\mathrm{w}}{\mathrm{d}^{\wedge} \mathrm{a}}+\frac{1-w}{\mathrm{~d}^{\wedge} \mathrm{b}}
$$

Where $\mathrm{R}$ is the resources allocated to the node, $\mathrm{d}$ is the distance from the node to the gateway, $\mathrm{d}>0, \mathrm{w}$ is the weight for each bias technique, $0<\mathrm{w}<1$, a, b are the biasing constants that determines the strength of each bias, $a, b>0$.

Of course this technique will not work as well when the entire network is completely congested, it depends on the assumption that at least some of the network has some free resources to make use of. However, this assumption is not unreasonable since one usually also assumes that a WMN can re-route packets through various links if one link has problems due to poor quality or over-use. For this assumption to be correct, this would mean that there must be some other link which can handle the extra capacity. This means that it is unlikely many of the solutions we have now would operate well in a WMN which is congested. Similarly to the distance biasing, we proposed to split the resources available to this scheme in half, and bias proportionally and strongly against the queue size. The queue size that we are referring to for this technique is the MAC layer queue. The formula which represents how resources are allocated with respect to queue size is seen in Equation 2

$$
\frac{1}{\mathrm{R}}=\frac{\mathrm{w}}{\mathrm{q}^{\wedge} \mathrm{a}}+\frac{1-w}{\mathrm{q}^{\wedge} \mathrm{b}}
$$

Where $\mathrm{R}$ is the resources allocated to the node, $\mathrm{q}$ is the length of the queue, $\mathrm{q}>0$, $w$ is the weight for each bias technique, $0<\mathrm{w}<$ $1, a, b$ are the biasing constants that determines the strength of each bias, $a, b>0$.

In this technique, we used the inverse of the normal resource allocation function because we want to give preference to those nodes which have the largest queues. This means that we waste less time giving resources to those nodes which do not have much to transfer in a given time. The mixed-bias technique, however, still gives some priority to those nodes which do have a small amount of traffic, so it will prevent starvation of these nodes.

If the link doesn't improve in quality, at least it is getting fewer opportunities to send packets, so the poor service the users will experience is related directly to the quality of their own link and does not negatively affect the rest of the network. In other schemes this might not always be the case. The poor quality link may try to communicate as if the link were behaving normally. Again we use the same biasing parameters in this scheme with a proportional bias (factor of 2) and a strong bias (factor of 5) mixed together.

$$
R=\frac{w}{q^{a} a}+\frac{1-w}{q^{A} b}
$$

Where $\mathrm{R}$ is the resources allocated to the node, $\mathrm{q}$ is the quality of the link $w$ is the weight for each bias technique, $0<\mathrm{w}<1$, a,b are the biasing constants that determines the strength of each bias, $a, b$ $>0$.

The key to the combined mixed-bias technique is providing a fraction of the resources to each of the mixed-bias techniques. For example, half the resources could be assigned to the distance technique while a quarter could be assigned to queue length and the last quarter to link quality. The network designer or administrator could specify these quantities manually. In a more complex network, these parameters could dynamically change depending on which applications are being run on the network at a given time or what the environment around the network is like.

\section{RESULTS}

In this paper, we implement fair scheduling and mixed-bias scheduling approach using OPPNET tool. There are several parameters used in this simulation. The main parameter settings are summarized in below Table.

\begin{tabular}{|c|c|}
\hline Parameter & Value \\
\hline Environment Dimension & $1000 \mathrm{X} 1000 \mathrm{~m}$ \\
\hline Node Range & $500 \mathrm{~m}$ \\
\hline Number of Mesh Router & 10 to 60 \\
\hline Number of Gateway & 1 to 6 \\
\hline Max. mode speed & $5 \mathrm{~m} / \mathrm{s}$ to $60 \mathrm{~m} / \mathrm{s}$ \\
\hline Data Packet Size & $28-1024$ bytes \\
\hline
\end{tabular}

The performance of the fair scheduling was evaluated using two simulation parameters. Our solution is compared to three different models: (i) model with no fair scheduling and a single gateway, (ii) no fair scheduling and multiple gateways and (iii) fair scheduling with a single gateway. We also separately compare the fair scheduling with a single gateway to that with multiple gateways to emphasize the benefit of using multiple gateways.

Figure 1 shows the average packet delivery ratio as a function of the number of mesh routers in the network. Results are plotted for the case with a single gateway and five gateways for both fair scheduling and no scheduling. As the network size increases, the difference between the techniques becomes more pronounced. The cases with multiple gateways have the highest packet delivery ratio.

Figure 2 shows the average packet delivery ratio as a function of the number of gateways in the network. These results were compiled with 60 mesh routers because larger network sizes are affected by a lack of gateways the most. Moreover, the results show performance improvement with fair scheduling with respect to average packet delivery ratio.

Figure 3 shows average end to end delays with varying mesh routers. These results were compiled with 60 mesh routers of single gateway no fair scheduling, six gateway no fair scheduling, proposed single gateway fair scheduling, proposed six gateway fair scheduling. The proposed single gateway fair scheduling, proposed 
six gateway fair scheduling both cases show better performance than no fair scheduling.

Table2: Average Packet Delivery Ratio for Single GW with Varying Mesh Routers 10 to 60

\begin{tabular}{|c|l|l|l|l|l|l|}
\hline $\begin{array}{c}\text { Number } \\
\text { Routers }\end{array}$ & $\mathbf{1 0}$ & $\mathbf{2 0}$ & $\mathbf{3 0}$ & $\mathbf{4 0}$ & $\mathbf{5 0}$ & $\mathbf{6 0}$ \\
\hline $\begin{array}{c}\text { Single GW } \\
\text { No Fair } \\
\text { Scheduling }\end{array}$ & 0.83 & 0.76 & 0.70 & 0.46 & 0.35 & 0.39 \\
\hline $\begin{array}{c}\text { Proposed } \\
\text { Single GW } \\
\text { Fair } \\
\text { Scheduling }\end{array}$ & 0.87 & 0.84 & 0.81 & 0.77 & 0.63 & 0.60 \\
\hline
\end{tabular}

Table 3: Average Packet Delivery Ratios with Varying

\begin{tabular}{|c|l|l|l|l|l|l|}
\hline Gateways $\mathbf{1}$ to 6 \\
\hline $\begin{array}{c}\text { Number of GW } \\
\begin{array}{c}\text { Average Packet } \\
\text { Delivery Ratios } \\
\text { No Fair } \\
\text { Scheduling }\end{array}\end{array}$ & $\mathbf{1}$ & $\mathbf{2}$ & $\mathbf{3}$ & $\mathbf{4}$ & $\mathbf{5}$ & $\mathbf{6}$ \\
\hline $\begin{array}{c}\text { Average Packet } \\
\text { Delivery Ratios } \\
\text { Proposed } \\
\text { Single GW } \\
\text { Fair } \\
\text { Scheduling }\end{array}$ & 0.53 & 0.64 & 0.71 & 0.77 & 0.83 & 0. \\
\hline
\end{tabular}

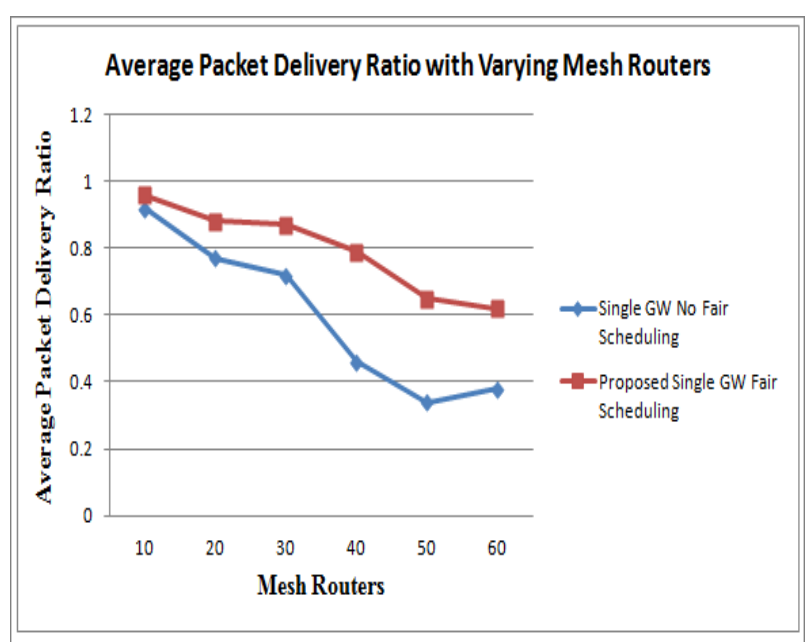

Figure 4 Average Packet Delivery Ratio with Varying Mesh Routers

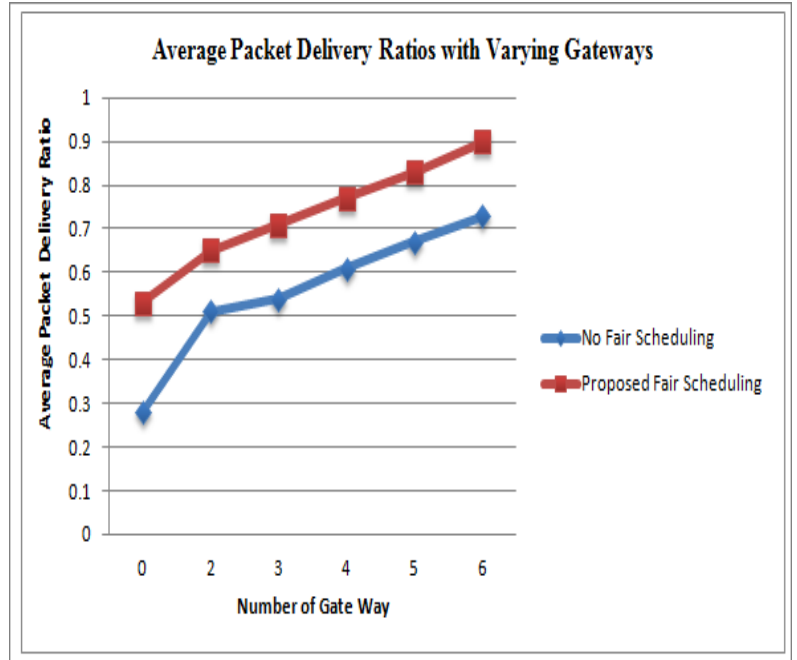

Figure 5 Average Packet Delivery Ratios with Varying Gateways

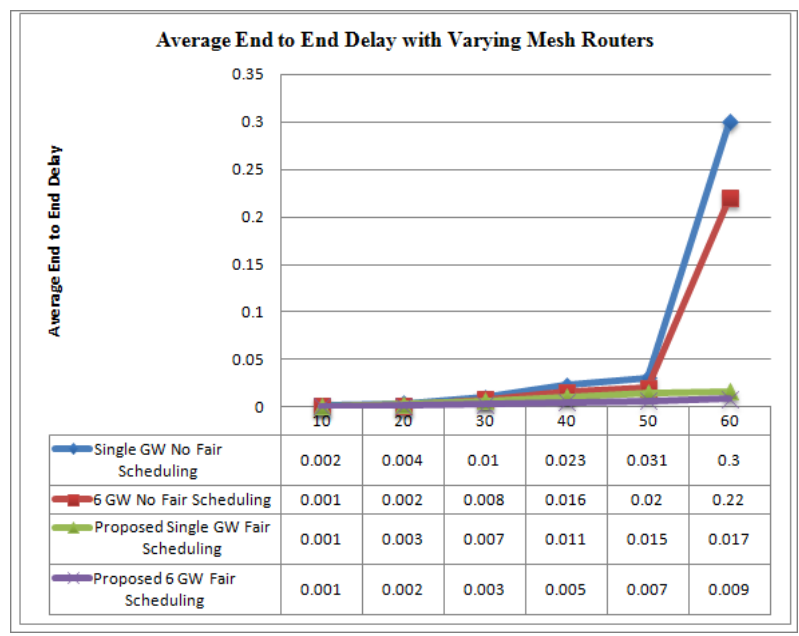

Figure 6 Average End to End Delays with Varying Mesh Routers

\section{CONCLUSION}

In this, two schemes were proposed for wireless mesh networks. First, the fair scheduling approach was compared against existing work with a single gateway and the results showed that using multiple gateways does indeed perform better than a single gateway in terms of packet delivery ratio and end-to-end delay. When compared against a solution that did not use any fair scheduling, the fair scheduling approach also performed better in terms of packet delivery ratio, however, in terms of delay it performed worse. This was likely due to the delay introduced by waiting to transmit only when no interference will be encountered. Moreover, due to the higher packet delivery ratio, more packets were dropped in the non fair scheduling approach causing lower delay overall. Second, the mixed-bias approaches we proposed were compared against IEEE 802.11 MAC in order to show that the mixed-bias approach is indeed a worthwhile scheduling approach for wireless mesh networks. Furthermore, we compared the original mixed bias distance approach against 802.11 MAC and our own approaches. All the experiments for mixed-bias evaluation were evaluated in terms of packet delivery ratio and average endto-end delay. The results provided verification for the original work since the mixed-bias distance approach performed better than 802.11 MAC in almost every case. Our proposed mixed-bias queue 
size approach performed better than the mixed-bias distance approach in most of the comparison scenarios.

\section{REFERENCES}

[1] Nessrine chakchouk and bechir hamdaoui, "traffic and interference aware scheduling for multiradio multichannel wireless mesh networks", ieee transactions on vehicular technology, vol. 60, no. 2, february 2011, pp 555-565.

[2] Erwu liu, qinqing zhang and kin k. Leung, "clique-based utility maximization in wireless mesh networks", ieee transactions on wireless communications 2011, pp 1-10.

[3] P.saravanaselvi and p.latha, " weighted fair queue scheduling algorithm for ieee802.16wireless networks", international conference on recent trends in computational methods, communication and controls (icon3c 2012) proceedings published in ijca, 2012, pp. 5-8.

[4] Yahya al-hazmi and hermann de meer, "virtualization of 802.11 interfaces for wireless mesh networks", ieee 2011 eighth international conference on wireless on-demand network systems and services, pp- 44-51.

[5] Ronghui hou, king-shan lui, fred baker, and jiandong li, "hop-by-hop routing in wireless mesh networks with bandwidth guarantees", ieee transactions on mobile computing-2011, pp-1-15.
[6] Yongsu gwak, jinsoo ahn and young yong kim, "network coding-based bridge routing wireless mesh network", ieee 2011(icoin), pp- 363-366.

[7] Jad el-najjar, hamed m.k. Alazemi, and chadi assi, "on the interplay between spatial reuse and network coding in wireless networks", ieee transactions on wireless communications, vol. 10 , no. 2 , february 2011 , pp- 560 569.

[8] Chetan kumar verma, bheemarjuna reddy tamma, b. S. Manoj, and ramesh rao, "a realistic small-world model for wireless mesh networks", ieee 2011.

[9] Jinyuan sun, chi zhang, yanchao zhang and yuguang fang, "sat: a security architecture achieving anonymity and traceability in wireless mesh networks", ieee transactions on dependable and secure computing, vol. 8 , no. 2, marchapril 2011, pp- 295-307.

[10] Yuefeng huang, xinyu yang, shuseng yang, wei yu, and xinwen fu, "a cross-layer approach handling link asymmetry for wireless mesh access networks", ieee transactions on vehicular technology, vol. 60 , no. 3 , march 2011, pp- 1045-1058. 